\title{
NEK2 regulates stem-like properties and predicts poor prognosis in hepatocellular carcinoma
}

\author{
SHUANG LIN ${ }^{1 *}$, SENJUN ZHOU $^{1 *}$, SHAOJIE JIANG $^{4}$, XIAOLONG LIU $^{1}$, YIFAN WANG ${ }^{1,2}$, \\ XUEYONG ZHENG ${ }^{1,2}$, HAIMENG ZHOU ${ }^{3}$, XUHUI LI $^{3}$ and XIUJUN CAI ${ }^{1,2}$ \\ ${ }^{1}$ Department of General Surgery, ${ }^{2}$ Key Laboratory of Laparoscopic Technology of Zhejiang Province, \\ Sir Run Run Shaw Hospital, College of Medicine, Zhejiang University, Hangzhou, Zhejiang; ${ }^{3}$ Zhejiang Provincial Key \\ Laboratory of Applied Enzymology, Yangtze Delta Region Institute of Tsinghua University, Jiaxing, Zhejiang; \\ ${ }^{4}$ Department of Radiology, Sir Run Run Shaw Hospital, College of Medicine, \\ Zhejiang University, Hangzhou, Zhejiang, P.R. China
}

Received January 20, 2016; Accepted April 21, 2016

DOI: $10.3892 / o r .2016 .4896$

\begin{abstract}
NEK2 has been estimated to play an important role in cancer progression. However, its relevance in hepatocellular carcinoma (HCC) has not yet been explored. Immunohistochemistry revealed NEK2 expression was upregulated in HCC. NEK2-positive hepatocellular carcinoma patients were associated with poor prognosis after surgery compared with NEK2-negative patients based on Kaplan-Meier curves. Deletion of NEK2 reduced self-renewal properties and chemotherapeutic resistance, and decreased the stemness associated genes in cell lines. NEK2 was associated with unfavorable outcomes in HCC patients, and was revealed to regulate self-renewal property by means of $\mathrm{Wnt} / \beta$-catenin signaling, and chemotherapeutic resistance by preferential regulation of the expression of $\mathrm{ABCG} 2$ and ALDH1A1 in HCC cells.
\end{abstract}

\section{Introduction}

Hepatocellular carcinoma (HCC) is one of the most common cancers and the third leading cause of cancer-related deaths in the world (1). Recently data showed that 782,500 new cases

Correspondence to: Professor Xiujun Cai, Department of General Surgery, Sir Run Run Shaw Hospital, College of Medicine, The Institute of Minimally Invasive Surgery, Zhejiang University, Hangzhou, Zhejiang 310016, P.R. China

E-mail: caixjzju@163.com

Dr Xuhui Li, Zhejiang Provincial Key Laboratory of Applied Enzymology, Yangtze Delta Region Institute of Tsinghua University, Jiaxing, Zhejiang, P.R. China

E-mail: lixuhui07@mails.tsinghua.edu.cn

${ }^{*}$ Contributed equally

Key words: NEK2, hepatocellular carcinoma, cancer stem cells, wnt/ $\beta$-catenin signaling and 745,500 deaths occurred worldwide during 2012, with China alone accounting for approximately $50 \%$ of the total number of cases and deaths (2). The majority of HCC occurs in the setting of chronic liver disease from viral hepatitis, alcohol abuse, heavy exposure to aflatoxin or algal hepatotoxins in contaminated water, betel nut chewing and diabetes mellitus (3). The process of HCC involves a series of sequential and complex steps. Over the past decades a large number of studies mostly focused on the cancer stem cells (CSCs).

CSCs are a subset of tumor cells that are capable of selfrenewal, chemo/radio-therapeutic resistance, tumorigenicity and differentiation, similar to normal stem cells $(4,5)$, and these characteristics could further result in aggressive phenotype of cancer and poor prognosis. Therefore, CSCs may serve as an effective therapeutic target in the treatment of HCC and may improve the current poor prognosis of this disease.

NIMA-related kinase 2 (NEK2), a member of the Nek family of serine/threonine kinases, is structurally related to the essential mitotic regulator NIMA and is highly enriched at the centrosome (6). Recent data indicate that NEK2 has emerged as an important player in cancer progression. Overexpression of NEK2 in myeloma (7), colorectal carcinomas $(8,9)$, breast carcinoma $(10,11)$, and lung cancer $(12)$ has been associated with aggressive disease, poor differentiation, development of metastases and poor clinical prognosis. Previous studies also revealed expression of Nek 2 and $\beta$-catenin were correlated with each other in clinical specimens of colorectal cancer and breast carcinoma $(8,10)$. Furthermore, $\beta$-catenin was proved as a Nek2 substrate involved in centrosome separation (13), and Nek 2 could bind to $\beta$-catenin to prevent its ubiquitination and degradation (14). $\beta$-catenin was well-known as a key components of canonical wnt/ $\beta$-catenin signal pathway which regulate the CSC features.

However, up to now, no data are available regarding the role of NEK2 in HCC and CSCs. In the present study, we evaluated that NEK2 is highly expressed in HCC, and associated with tumor recurrence and poor prognosis. Furthermore, this study also revealed the role of NEK2 in CSCs, including maintaining self-renewal property by means of $\mathrm{Wnt} / \beta$-catenin signaling, 
and influencing chemotherapeutic resistance by preferential regulating the expression of ABCG2 and ALDH1A1 in HCC cells.

\section{Materials and methods}

Patients and specimens. The tumor tissues with adjacent nontumor tissues were from 40 patients, and tumor specimens with clinicopathological features and follow-up, were obtained in 104 patients. All patients underwent curative hepatectomy for HCC at Sir Run Run Shaw Hospital, College of Medicine, Zhejiang University, from January 2006 to December 2010. All the cases conformed to the following criteria: diagnosed by postoperative histopathology, no perioperative extrahepatic metastasis, no other malignant diseases, did not die from perioperative complications and no preoperative anticancer therapy. Curative resection was defined as removal of all recognizable tumors with a clear microscopic margin. Follow-up data were recorded from the patient's medical records and completed by a telephone survey. All subjects selected were required to provide written informed consent on the use of clinical specimens for medical research. The study was approved by the Ethics Committee of our hospital.

Cell lines and cell culture. Normal human liver cell line (Chang liver) and HCC cell lines Hep-G2, Hep-3B, HuH-7, SMMC7721, HCCLM3, Snu387 and Snu475 were purchased from the American Type Culture Collection (ATCC; Manassas, VA, USA) or the Cell Bank of Shanghai Institutes for Biological Sciences, the Chinese Academy of Sciences (Shanghai, China). Chang liver, HuH-7, SMMC7721 and HCCLM3 were routinely maintained in Dulbecco's modified Eagle's medium (DMEM). Hep-G2 and Hep-3B were cultured in Minimum Essential Medium (MEM). Snu387 and Snu475 were in RPMI-1640 medium. All cell lines were supplemented with $10 \%$ fetal bovine serum (FBS; Gibco-Invitrogen Carlsbad, CA, USA), 100 units $/ \mathrm{ml}$ penicillin, and $100 \mathrm{mg} / \mathrm{ml}$ streptomycin at $37^{\circ} \mathrm{C}$ in a humidified incubator under $5 \% \mathrm{CO}_{2}$.

Immunohistochemistry (IHC) and IHC evaluation. Immunohistochemical stainings were performed following standard procedure. Briefly, formalin-fixed and paraffin embedded human samples were first cut into $5-\mu \mathrm{m}$-thick sections. Then the antigen retrieval was accomplished by deparaffinization, rehydration, and boiling in a microwave oven with citrated buffer. Hydrogen peroxide (3\%) in PBS was used to block the endogenous peroxidase activity and BSA was used to block non-specific staining. Sections were incubated with NEK2 antibody (AP8074c; Abgent, San Diego, CA, USA) and $\beta$-catenin antibody (\#9582; Cell Signaling Technology, Inc., Danvers, MA, USA) at $4^{\circ} \mathrm{C}$ overnight. The EnVision kit (Dako, Carpinteria, CA, USA) was used to detect primary antibody followed by staining with DAB reagent and counterstaining with hematoxylin.

To evaluate IHC staining of NEK $2 / \beta$-catenin in the nuclear and cytoplasmic regions, the expression of NEK2/ $\beta$-catenin was scored as absent staining (-), weak staining $(+)$, moderate staining $(++)$ and strong staining $(+++)$. In the present study, we characterize a low $(-/+)$ score of NEK $2 / \beta$-catenin as 'NEK2/ $\beta$-catenin negative' and a high $(++/+++)$ score of
NEK $2 / \beta$-catenin as 'NEK $2 / \beta$-catenin positive', respectively $(15,16)$. Assessments of the staining were scored in a double-blinded manner by two experienced pathologists. When a discrepancy arose for any case, the two pathologists discussed it and reached the final score by consensus.

Protein extraction and western blot analysis. Cell lysates were generated and total proteins were separated by standard SDS-PAGE, followed by transfer to PVDF membranes. The membranes were then washed and blocked before incubation of primary antibody (NEK-2, AP8074c, Abgent; $\beta$-catenin, \#9582, Cell Signaling Technology; Bim1,\#6964,Cell Signaling Technology; Sox 2, \#3579, Cell Signaling Technology; Nanog, AP21336c, Abgent; and $\beta$-actin, BS6007M, Bioworld Technology, St. Louis Park, MN, USA), followed by incubation of horseradish peroxidase (HRP)-conjugated secondary antibodies. The reactions were detected by enhanced chemiluminescence assay. $\beta$-actin was used as control.

$R N A$ preparation and quantitative real-time PCR. All procedures were performed according to the manufacturer's instructions. Total RNA was extracted using the Ultrapure RNA kit (CWbio, Co., Ltd., Beijing, China). RNA was reverse transcribed into cDNA using iScript cDNA Synthesis kits (Bio-Rad Laboratories, Hercules, CA, USA). Quantitative real-time PCR (qRT-PCR) was performed using SYBR-Green PCR kit (Applied Biosystems). GADPH was used as loading control. Specific primers for the amplification of target genes and GAPDH were listed in Table I.

Small interfering RNA and transfection. NEK2 siRNA sequences were previously performed (17). The NEK2 siRNANEK2 sequence were 5'-UGCACUUGGACUUAGAUGUG AGCUG-3' (sence) and 5'-CAGCACAUCUAAGUCCAAGU GCA-3' (antisence). The NEK2 siRNA-control sequences were 5'-UUCUCCGAACGUGUCACGUTT-3' (sence) and 5'-ACGUGACACGUUCGGAGAATT-3' (antisence).

Transfection of the siRNAs for HCC cells was performed with Lipofectamine 3000 (Invitrogen) according to the manufacturer's instructions. After $48 \mathrm{~h}$ of transfection, cells were lysed for western blot analysis and quantitative real-time PCR. For chemosensitivity, colony formation and spheroid formation assay, cells were collected $24 \mathrm{~h}$ after transfection.

Spheroid formation assay, colony formation assay and chemosensitivity assay. For spheroid formation assays, single cell suspensions of $2 \times 10^{4}$ cells were seeded in 6-well ultra-low attachment plates (Corning, Inc., Corning, New York, USA) in complete mammoCult ${ }^{\mathrm{TM}}$ medium (Stem Cell Technologies, Vancouver, BC, Canada). Cells were cultured in mammoCult media according to the manufacturer's instructions. The number of spheres for each well was evaluated after 7 days of culture.

For colony formation assays, cells were seeded in a 6-well plate $\left(1 \times 10^{3}\right.$ cells/well). After incubation at $37^{\circ} \mathrm{C}$ for 7 days, the cells were washed twice with PBS and stained with $0.1 \%$ crystal violet solution. The dishes were photographed and the colonies were counted.

Chemosensitivity assay was determined by Cell Counting kit-8 (CCK-8; Dojindo Laboratories, Kumamoto, Japan). 
Table I. Sequences of gene-specific primers used for real-time RT-PCR.

\begin{tabular}{lll}
\hline Gene & \multicolumn{1}{c}{ Primer sequence forward $\left(5^{\prime}-3\right.$ ') } & Primer sequence reverse $\left(5^{\prime}-3 '\right)$ \\
\hline NEK2 & CATTGGCACAGGCTCCTAC & GAGCCATAGTCAAGTTCTTTCCA \\
ABCG2 & CACCTTATTGGCCTCAGGAA & CCTGCTTGGAAGGCTCTATG \\
ALDHIA1 & TGGAATGTGGAGGAGGCCCGT & CACCAAAGGGGCACTGGGCA \\
$\beta$-catenin & GTCTTACCTGGACTCTGGAATCC & GGTATCCACATCCTCTTCCTCAG \\
E-Myc & GGCTCCTGGCAAAAGGTCA & CTGCGTAGTTGTGCTGATGT \\
CD133 & TAATCGTCAATGCCAGTGTACTTC & CTTCTCCCAAGTTTTGAGCCAT \\
K19 & TGGATGCAGAACTTGACAACGT & ATACCTGCTACGACAGTCGTGGT \\
LIN28 & TTTGAGACGGAACAGGCTCT & TCAGTAACCTCGGACCTGCT \\
NOTCH1 & TGTAAGTGGTTCAACGTGCG & CCTCACCCTCCTTCAAGCTC \\
GAPDH & GAGGCGTGGCAGACTATGC & CTTGTACTCCGTCAGCGTGA \\
\hline
\end{tabular}
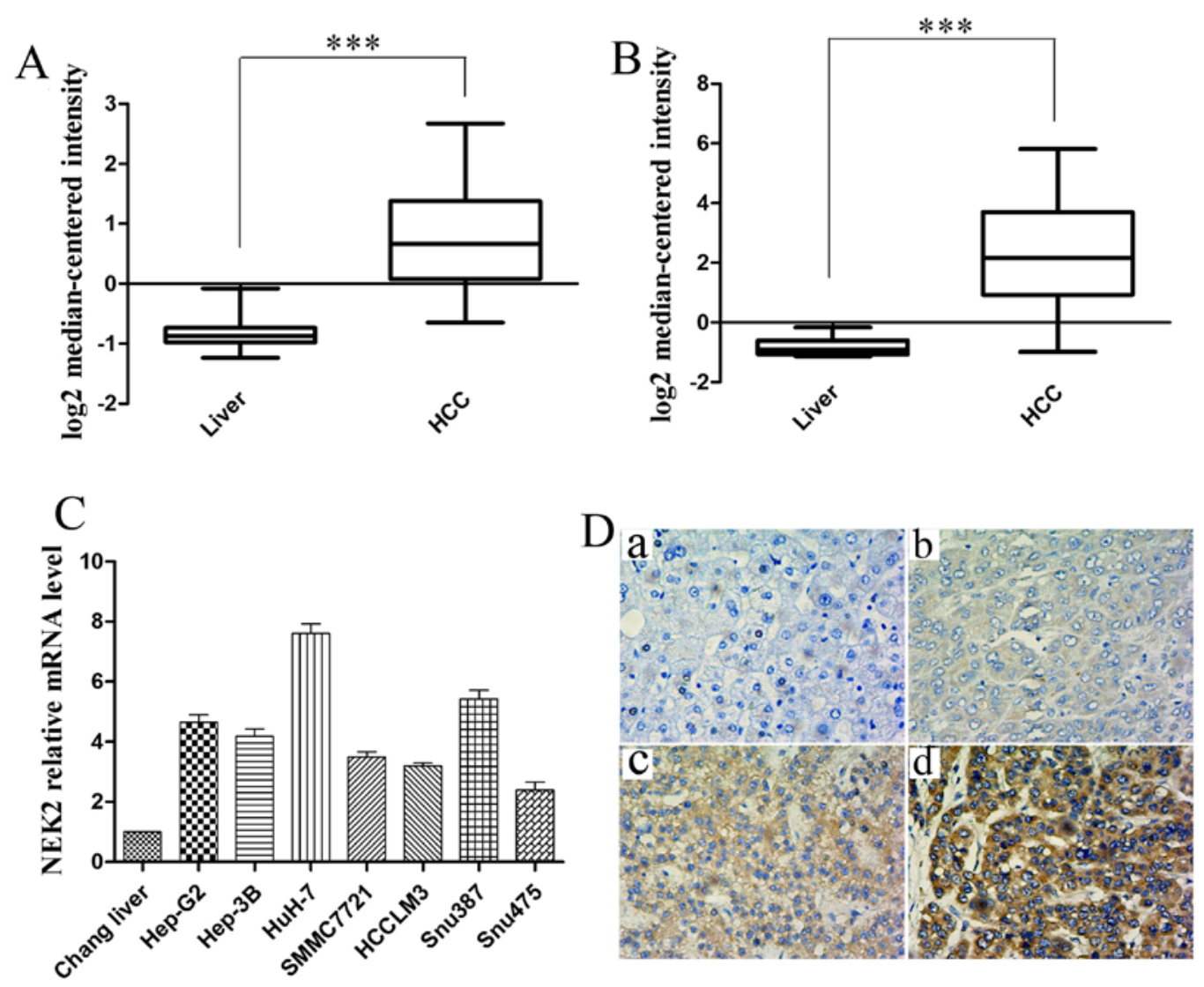

Figure 1. NEK2 expression is upregulated in HCC. (A and B) Analysis from the Oncomine database shows that mRNA expression levels of NEK2 is significantly higher in HCC compared to normal liver tissues. Data were pooled together from two published HCC gene expression studies Wurmbach Liver Statistics (A), Hepatology 2007/04/01 and Roessler Liver Statistics (B), Cancer Res 2010/12/15 (all P<0.001). (C) The mRNA level of NEK2 in Chang liver and $7 \mathrm{HCC}$ cell lines with different differentiation status. Data were normalized against the NEK2 expression level in Chang liver cells. (D) Immunohistochemical staining of NEK2 expression in human HCC specimens and their matched normal tissues. Negative (-) (a) or weak (+) (b) staining were defined as negative expression; moderate (++) (c) or strong (+++) (d) staining were defined as positive expression. Original magnification, $\mathrm{x} 200$.

Briefly, 6x10³ cells in $100 \mu 1$ medium was dispensed into a 96-well plate. After overnight incubation, cells were exposed to 5-fluorouracil (5-Fu) and cisplatin for $72 \mathrm{~h}$, respectively. Then CCK-8 was added to the wells and incubated for $1 \mathrm{~h}$. Finally, the absorbance of the sample taken from each well was detected at $450 \mathrm{~nm}$.
Statistical analysis. The statistical analyses were performed using GraphPad Prism software version 5.0 (GraphPad Software, Inc., La Jolla, CA, USA) and SPSS version 19.0 (SPSS, Inc., Chicago, IL, USA). A statistical analysis for group differences was performed by using the Chi-square test or the Student's t-test. The mean SD of three independent 
Table II. Relationship between NEK2 expression and clinicopathological features.

\begin{tabular}{|c|c|c|c|}
\hline \multirow[b]{2}{*}{ Characteristics } & \multicolumn{2}{|c|}{$\begin{array}{c}\text { NEK2 expression } \\
\mathrm{n}(\%)\end{array}$} & \multirow[b]{2}{*}{ P-value } \\
\hline & $\begin{array}{l}\text { Negative } \\
\text { (44) }\end{array}$ & $\begin{array}{l}\text { Positive } \\
\text { (60) }\end{array}$ & \\
\hline Age (years) & & & 0.539 \\
\hline$\leq 50$ & 23 & 35 & \\
\hline$>50$ & 21 & 25 & \\
\hline Gender & & & 0.123 \\
\hline Male & 32 & 51 & \\
\hline Female & 12 & 9 & \\
\hline Differentiation & & & 0.572 \\
\hline Well/medium & 21 & 32 & \\
\hline Poorly/undifferentiated & 23 & 28 & \\
\hline Cirrhosis & & & 0.656 \\
\hline Absent & 21 & 26 & \\
\hline Present & 23 & 34 & \\
\hline Tumor number & & & 0.286 \\
\hline Single & 41 & 52 & \\
\hline Multiple & 3 & 8 & \\
\hline Tumor size $(\mathrm{cm})$ & & & 0.539 \\
\hline$\leq 5$ & 29 & 36 & \\
\hline$>5$ & 15 & 24 & \\
\hline TNM stage & & & 0.262 \\
\hline I & 37 & 45 & \\
\hline II/III & 7 & 15 & \\
\hline BCLC stage & & & 0.945 \\
\hline A & 12 & 16 & \\
\hline $\mathrm{B}$ & 32 & 44 & \\
\hline PVTT & & & 0.764 \\
\hline Absent & 39 & 52 & \\
\hline Present & 5 & 8 & \\
\hline$\beta$-catenin expression & & & $<0.001$ \\
\hline Positive & 23 & 11 & \\
\hline Negative & 21 & 49 & \\
\hline
\end{tabular}

PVTT, portal vein tumor thrombus.

experiments is reported. Recurrence free survival (RFS) and overall survival (OS) after the operation were calculated using the Kaplan-Meier method. Multivariate analysis of prognostic factors for survival was performed by a Cox stepwise regression model. A P-value of $<0.05$ was considered to be statistically significant.

\section{Results}

NEK2 expression is upregulated in HCC. To determine the significance of NEK2 in HCC, we first analyzed multiple microarray data sets in the Oncomine database (www.onco-
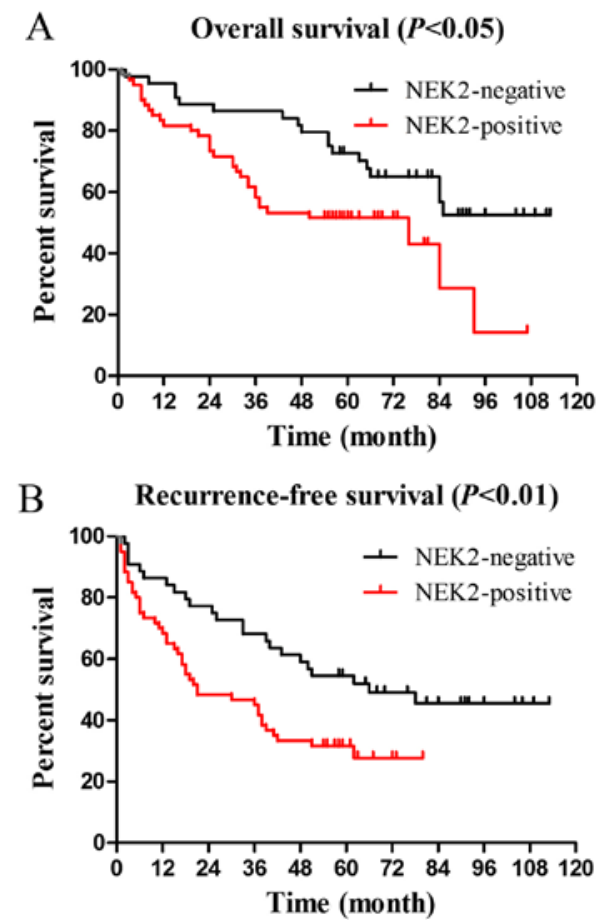

Figure 2. Correlation between NEK2 expression and clinical outcome. (A and B) Kaplan-Meier survival analysis of NEK2 expression in HCC patients. Kaplan-Meier OS (A, $\mathrm{P}<0.05)$ and RFS $(\mathrm{B}, \mathrm{P}<0.01)$ survival curves of $104 \mathrm{HCC}$ patients in association with NEK2 expression. The OS and RFS rates were significantly decreased in NEK2-positive HCC patients $(n=60)$ compared with NEK2-negative HCC patients $(n=44)$.

mine.com). As showed in Fig. 1A and B, we found NEK2 mRNA levels were significantly increased in HCC samples as compared to normal liver tissue from two published HCC gene expression studies Wurmbach Liver Statistics (18) and Roessler Liver Statistics (19) (all P $<0.001)$. We further assessed the mRNA expression of NEK2 in multiple $\mathrm{HCC}$ cell lines by real-time RT-PCR test. As shown in Fig. 1C, the mRNA levels of NEK2 were higher in the seven HCC cell lines including Hep-G2, Hep-3B, HuH-7, SMMC7721, HCCLM3, Snu387 and Snu475 than that in Chang liver.

To verify the microarray analysis results, we performed IHC experiments on 40 pairs human HCC specimens and their matched normal tissues. Immunohistochemical analysis showed NEK2 positive staining was detected in the nuclear and cytoplasmic regions. The expression of NEK2 was classified into negative (-) or weak positive ( + ) and moderate positive $(++)$ or strong positive (+++) staining in Fig. 1D. Positive staining of NEK2 could be observed in 23 of 40 (57.5\%) cases of HCCs, whereas NEK 2 showed positive staining in only 3 of $40(7.5 \%)$ cases of adjacent non-tumor tissues.

Correlation between NEK2 expression and clinical outcome. To better understand the clinical significance of NEK 2 expression in HCC, we investigated the clinicopathological features of NEK2 in 104 HCC samples. As shown in Table II, a positive NEK2 protein level was significantly associated with $\beta$-catenin expression $(\mathrm{P}<0.001)$. In contrast, NEK2 expression was not correlated with age, gender, HBsAg, differentiation, tumor number, tumor size, TNM stage, BCLC stage and portal vein tumor thrombus (PVTT) (all P>0.05). 

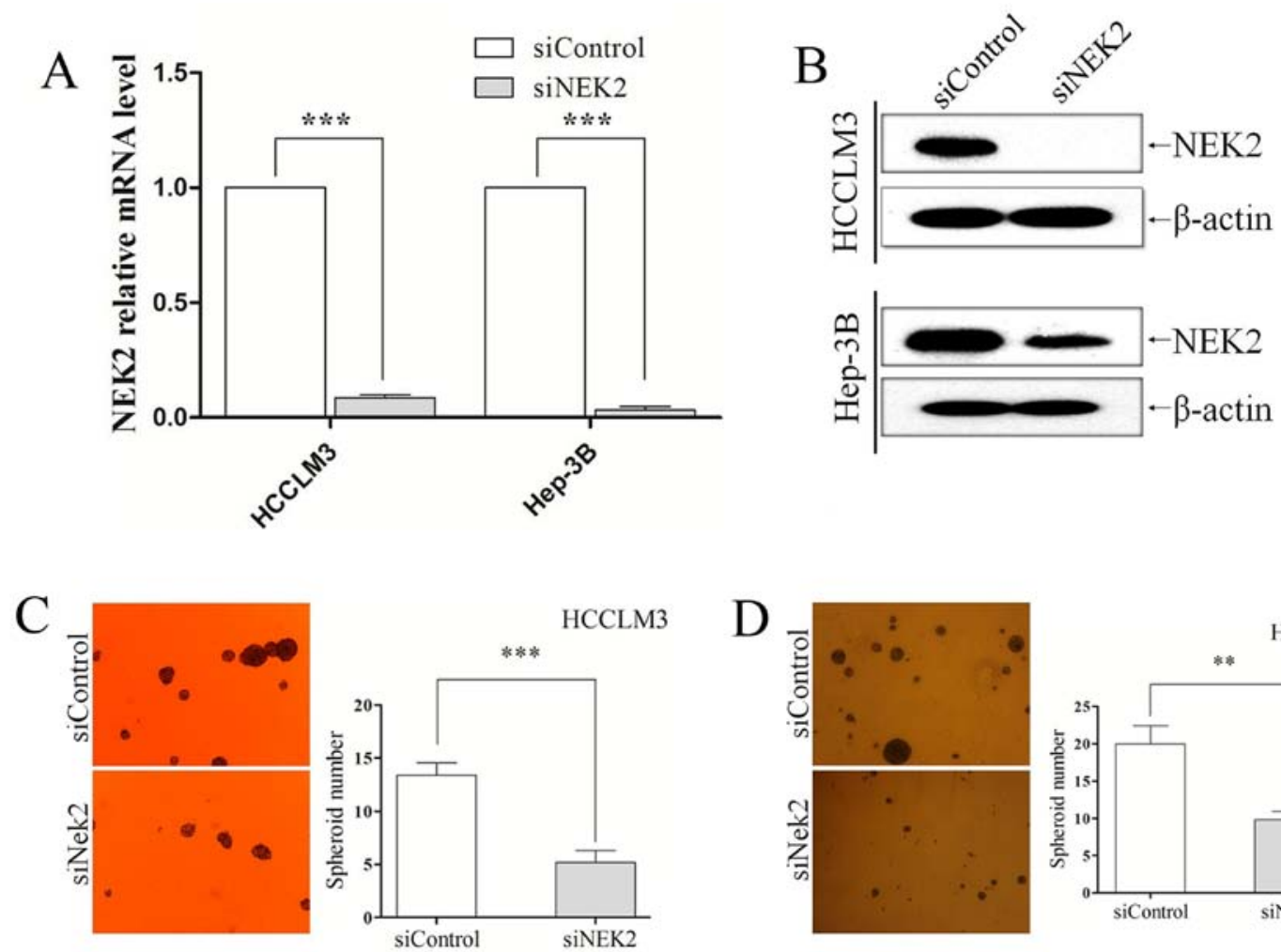

$\mathrm{D}$
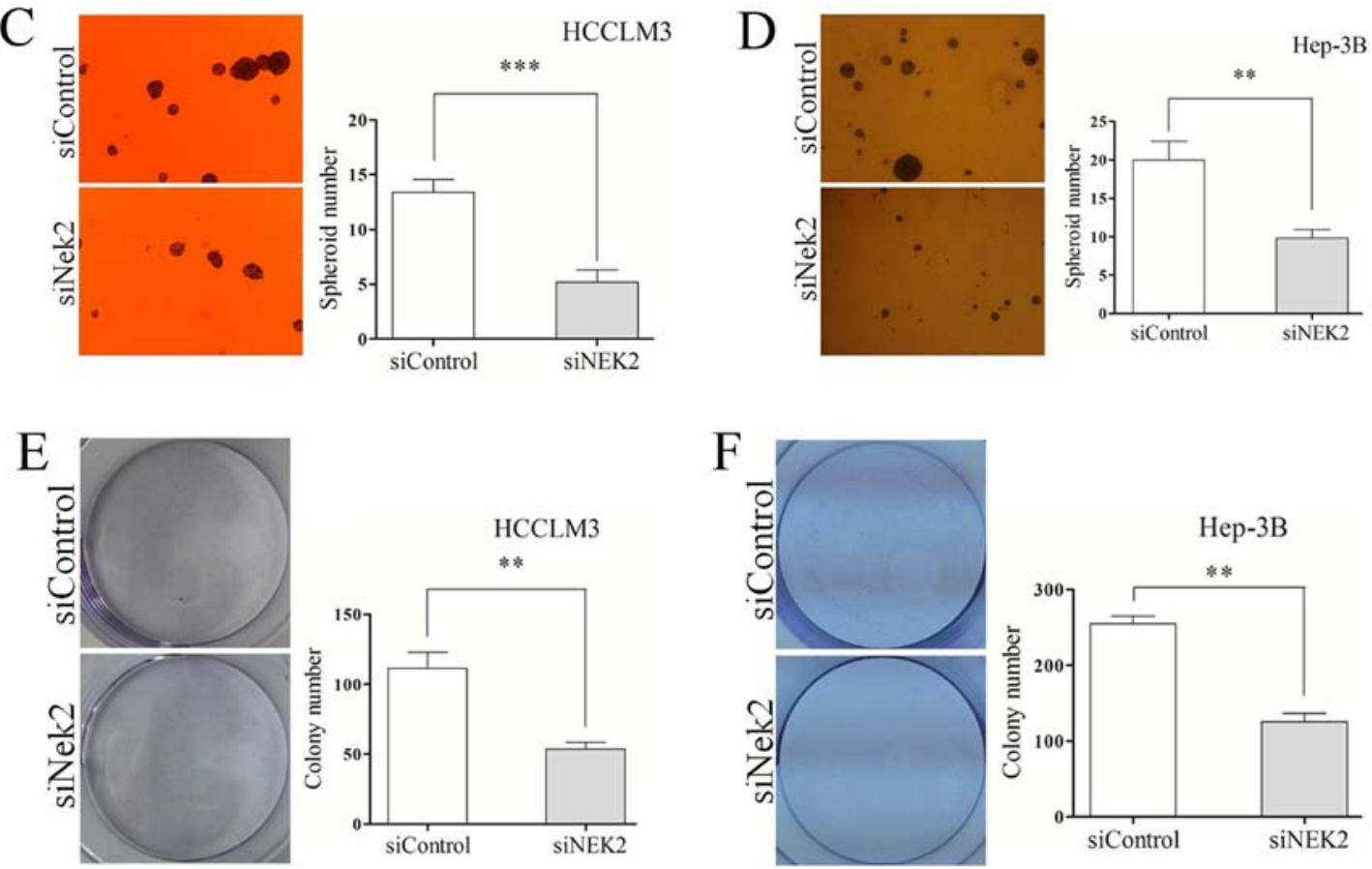

Figure 3. NEK2 knockdown decreased the self-renewal property of CSCs. (A) siRNA against NEK2 effectively decreased NEK2 expression verified by qRTPCR (all P<0.001). (B) siRNA against NEK2 effectively decreased NEK2 expression detected by western blotting. (C and D) siRNA-NEK2 cells generated a decreased number of spheroid formation compared with siRNA-Control cells. Representative phase-contrast image of HCC spheroids derived from (C) HCCLM3 and (D) Hep-3B cells are shown; (all P<0.01). NEK2 knockdown reduced the number of colony formation in both (E) HCCLM3 and (F) Hep-3B cell lines. (all $\mathrm{P}<0.01)$

To assess the prognostic significance of NEK2 expression, Kaplan-Meier curves for OS and RFS were analyzed. The OS and RFS rates at 5 years were 51.7 and $31.7 \%$ for NEK2 positive patients $(\mathrm{n}=60)$ compared with 72.7 and $54.5 \%$ for NEK2 negative patients $(\mathrm{n}=44)$, respectively (OS $\mathrm{P}<0.05$, Fig. 2A; RFS P $<0.01$, Fig. 2B).

Univariate Cox regression analysis was conducted to identify important prognostic factors of OS. NEK2 expression $(\mathrm{P}=0.014)$, $\mathrm{PVTT}(\mathrm{P}<0.001)$, TNM stage $(\mathrm{P}<0.001)$ and tumor size $(\mathrm{P}=0.034)$ were identified as important risk factors for OS (Table III). However, in multivariate Cox analysis, NEK2 expression $(\mathrm{P}=0.033)$ and tumor size $(\mathrm{P}=0.032)$ were found to be independent negative prognostic factors for OS (Table III).

These results indicated that the positive expression of NEK2 was associated with unfavorable outcomes in HCC patients.
NEK2 knockdown decreases the self-renewal property of CSCs. $\beta$-catenin is a key downstream molecule in the Wnt/ $\beta$-catenin signaling pathway, which is one of the wellknown pathways to play a critical role in CSC formation and maintenance $(20,21)$. The results from the clinical samples indicated a correlation between NEK 2 levels and $\beta$-catenin, so we next investigated whether NEK2 knockdown influenced the stemness characteristics of CSCs in HCC. Given that selfrenewal is a hallmark of CSCs, we performed sphere forming assay and colony formation assay to investigate the role of NEK2 in maintaining self-renewal property in HCC cells.

siRNA technology was used to knockdown NEK2, and its levels were effectively downregulated in HCCLM3 and Hep-3B, verified by qRT-PCR (all P<0.001; Fig. 3A) and western blotting (Fig. 3B). In the sphere formation assay, we found that NEK2 knockdown group and control group grew 
Table III. Univariate and multivariate Cox regression analysis for OS (HR hazard ratio, CI confidence interval).

\begin{tabular}{|c|c|c|c|c|}
\hline \multirow[b]{3}{*}{ Variables } & \multicolumn{4}{|c|}{ OS } \\
\hline & \multicolumn{2}{|c|}{ Univariate analysis } & \multicolumn{2}{|c|}{ Multivariate analysis } \\
\hline & $\mathrm{HR}(95 \% \mathrm{CI})$ & P-value & $\mathrm{HR}(95 \% \mathrm{CI})$ & P-value \\
\hline Age (years) ( $\leq 50$ vs. $>50)$ & $0.981(0.559-1.720)$ & 0.946 & $1.217(0.657-2.254)$ & 0.533 \\
\hline Gender (Male vs. female) & $1.213(0.619-2.376)$ & 0.573 & $1.511(0.728-3.134)$ & 0.268 \\
\hline Cirrhosis ( Absent vs. present) & $1.627(0.911-2.908)$ & 0.100 & $1.872(0.998-3.512)$ & 0.051 \\
\hline Tumor number (Single vs. multiple) & $1.384(0.588-3.255)$ & 0.457 & $0.538(0.129-2.246)$ & 0.395 \\
\hline Tumor size ( $\leq 5$ vs. $>5 \mathrm{~cm})$ & $1.827(1.046-3.191)$ & 0.034 & $2.140(1.069-4.283)$ & 0.032 \\
\hline TNM stage (I vs. II/III) & $3.129(1.707-5.735)$ & $<0.001$ & $2.311(0.414-12.885)$ & 0.339 \\
\hline BCLC stage (A vs. B) & $1.566(0.801-3.063)$ & 0.190 & $0.998(0.432-2.303)$ & 0.996 \\
\hline \multicolumn{5}{|l|}{ Differentiation } \\
\hline (Well/medium vs. poorly/undifferentiated) & $1.285(0.738-2.240)$ & 0.376 & $0.673(0.352-1.286)$ & 0.230 \\
\hline PVTT (Absent vs. present) & $5.765(2.925-11.364)$ & $<0.001$ & $3.569(0.734-17.365)$ & 0.115 \\
\hline NEK2 expression (Positive vs. negative) & $2.122(1.162-3.875)$ & 0.014 & $1.984(1.058-3.719)$ & 0.033 \\
\hline
\end{tabular}

PVTT, portal vein tumor thrombus.
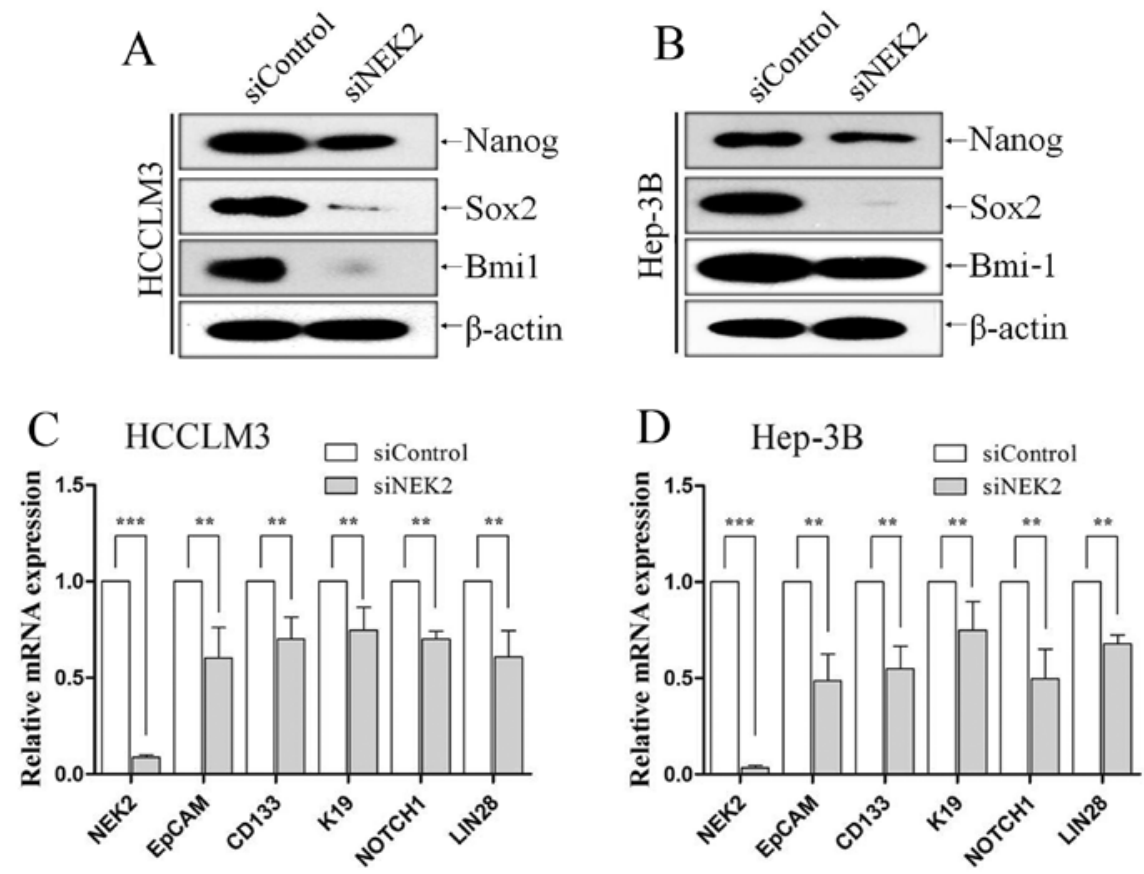

Figure 4. Deletion of NEK2 reduced the expression of stemness associated genes of CSCs. Western blot analysis was performed for Bmi-1, Sox 2 and Nanog in (A) HCCLM3 and (B) Hep-3B cells transfected with siControl or siNEK2. QRT-PCR was performed using primers for EpCAM, CD133, K19, NOTCH1 and LIN28 in (C) HCCLM3 and (D) Hep-3B cells transfected with siControl or siNEK2; (all P<0.01).

in the form of suspended individual cells on the first day. As 7 days passed, HCCLM3 and Hep-3B cells in which NEK2 expression was knocked down exhibited fewer and smaller spheres (all $\mathrm{P}<0.01$; Fig. $3 \mathrm{C}$ and D). Colony formation assay showed that NEK2 deletion formed less and smaller colonies than their control groups. NEK2 knocked-down also resulted in generation of a decreased ability to form colonies in HCCLM3 and Hep-3B cells (all P<0.01; Fig. 3E and F).

Deletion of NEK2 reduces the expression of stemness associated genes of CSCs. NEK2 had been proved to influence self-renewal of HCC CSCs, then we investigate whether the knockdown of NEK 2 suppresses stemness associated genes by means of western blot analysis and qRT-PCR. We investigated whether NEK2 influences the expression of transcriptional factors including Bmi-1, Sox 2 and Nanog, which are essential for maintaining stem cell phenotypes. Compared with the control group, western blot analysis showed NEK2 knockdown decreased the levels of stem cell genes Bmi-1, Sox2 and Nanog in HCCLM3 (Fig. 4A) and Hep-3B (Fig. 4B) cells. Additionally, the expression levels of the hepatic CSC markers EpCAM, CD133, K19, LIN28 and NOTCH1 were analyzed 
A

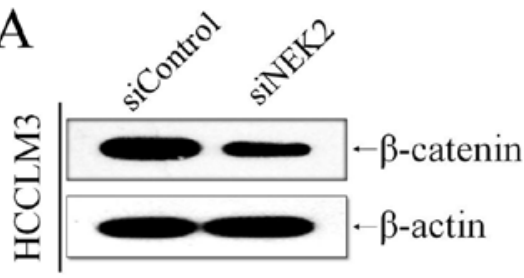

B

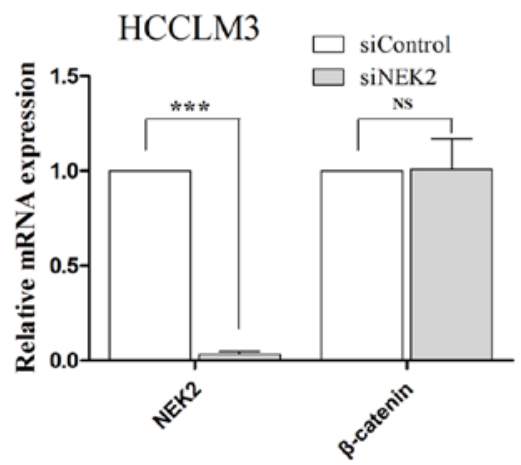

C

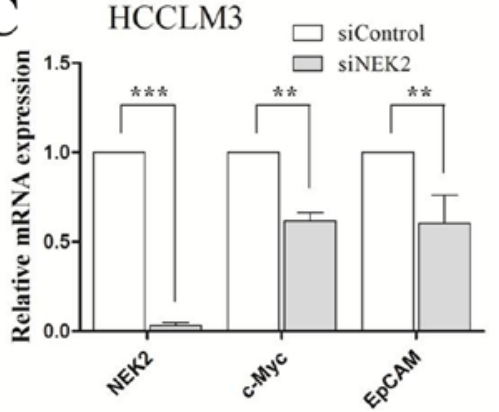

D

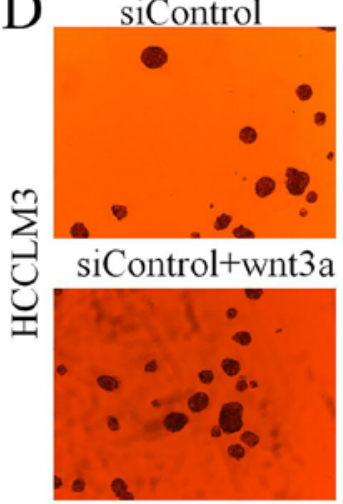

E

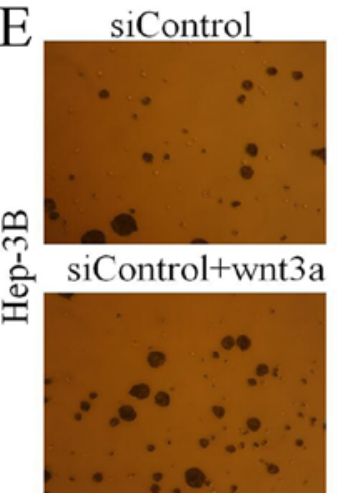

siNEK2
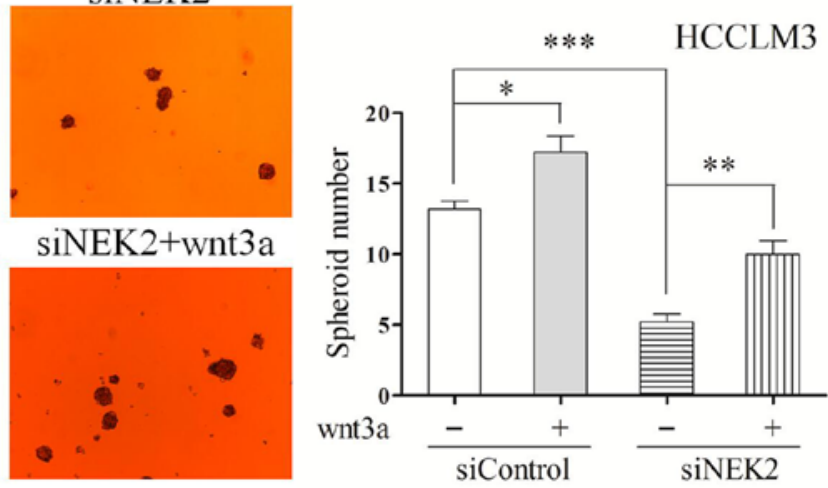

siNEK2

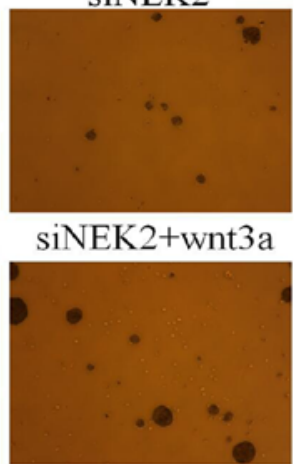

Figure 5. NEK2 regulates the self-renewal property of CSCs through the canonical wnt/ $\beta$-catenin signal pathway. (A) Western blot analysis was performed for $\beta$-catenin in HCCLM3 and Hep-3B cells transfected with siControl or siNEK2. (B) QRT-PCR was performed using primers for $\beta$-catenin in HCC cells transfected with siControl or siNEK2; (all P<0.01). (C) QRT-PCR was performed using primers for c-Myc and EpCAM in HCCLM3 and Hep-3B cells transfected with siControl or siNEK2; (all $\mathrm{P}<0.01)$. (D and E) Wnt3a $(20 \mathrm{ng} / \mathrm{ml}$ ) was added in cultures after cells were transfected with siRNA for $24 \mathrm{~h}$ : Wnt3a addition increased the ability of tumorsphere formation to partially compensate NEK2 inhibition in HCCLM3 (P<0.01; D) and Hep-3B (P<0.01; E). 

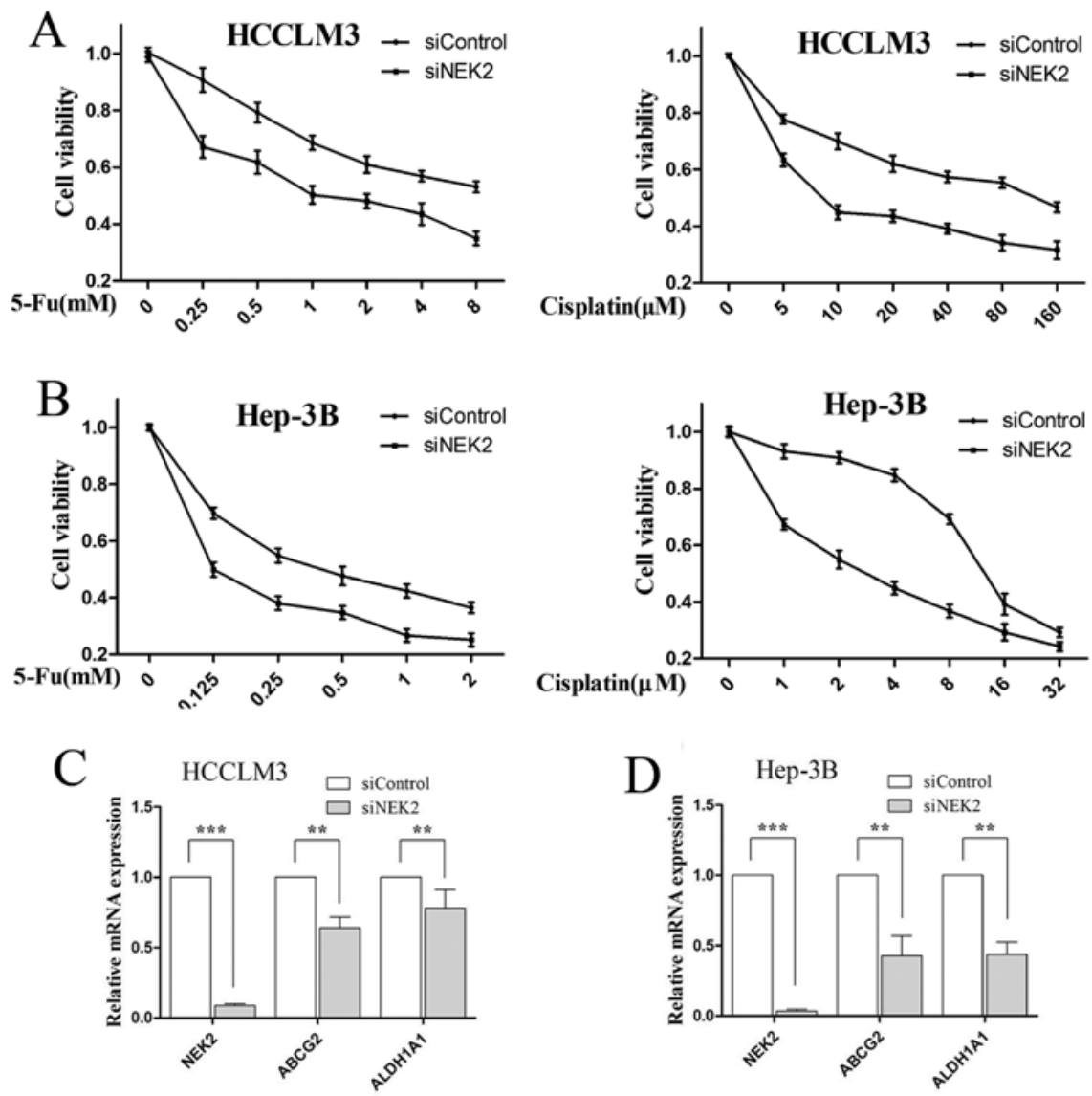

Figure 6. NEK2 deletion reduces chemotherapeutic resistance of CSCs. Knockdown of NEK2 resulted in reduced chemosensitivity after treatment with chemotherapeutic agents 5-Fu and cisplatin for $72 \mathrm{~h}$ in (A) HCCLM3 and (B) Hep-3B cells. QRT-PCR analysis of ABCG2 and ALDH1A1 in (C) HCCLM3 and (D) Hep-3B cells transfected with siControl or siNEK2; (all $\mathrm{P}<0.01$ ).

by qRT-PCR. All of these markers have been reported to be enriched in hepatic CSCs. QRT-PCR analysis also revealed that NEK2 deletion in HCCLM3 and Hep-3B cells expressed lower mRNA levels of EpCAM, CD133, K19, LIN28 and NOTCH1 (all $\mathrm{P}<0.01$; Fig. 4C and D).

$N E K 2$ regulates the self-renewal property of CSCs through the canonical wnt/ $\beta$-catenin signal pathway. Next, we explored the molecular mechanisms by which NEK2 affects the self-renewal traits of CSCs. Recent studies indicate that Wnt/ $\beta$-catenin pathway play a key role in hepatic CSCs (22) and its deregulation has been extensively reported in HCC $(23,24)$. We examined both expression of the NEK2 and $\beta$-catenin from previous HCC specimens by IHC. Nek 2 positive expression was found to associate with $\beta$-catenin positive expression $(\mathrm{r}=0.358, \mathrm{P}<0.001$; Table III). Western blot analysis also showed NEK 2 knockdown reduced $\beta$-catenin protein (Fig. 5A), but the mRNA level of $\beta$-catenin was not altered $(\mathrm{P}<0.01$; Fig. 5B) in HCC cells, which meant NEK2 regulated $\beta$-catenin through post-translational modification. This result was in accordance with that previously shown, i.e. NEK2 binds to $\beta$-catenin to prevent its ubiquitination and degradation (14). Subsequently, we used qRT-PCR to assessed the expression of downstream target genes of wnt/ $\beta$-catenin signal pathway including EpCAM and c-Myc. The expression of EpCAM and c-Myc in NEK2 knockdown group was significantly decreased compared with controls (all $\mathrm{P}<0.01$; Fig. 5C).
Wnt3a, a canonical Wnt ligand, activates the canonical Wnt signaling pathway $(25,26)$. Moreover, to confirm that the self-renewal effect of NEK2 was caused by activation of the Wnt/ $\beta$-catenin pathway, we enhanced canonical Wnt signaling with exogenous recombinant human Wnt3A (R\&D Systems, Minneapolis, MN, USA; $20 \mathrm{ng} / \mathrm{ml}$ ). HCC cells were transfected with siRNA for $24 \mathrm{~h}$, subsequently were cultured in the presence of recombinant Wnt3a. As confirmed, NEK2 knockdown reduced the ability to form tumorspheres in vitro, conversely, Wnt3a addition increased the ability of tumorsphere formation to partially compensate NEK2 inhibition in HCCLM3 (all $\mathrm{P}<0.01$; Fig. 5D) and Hep-3B (all $\mathrm{P}<0.01$; Fig. 5E). All these results demonstrate the important role of NEK2 in maintaining self-renewal property by means of Wnt/ $\beta$-catenin signaling.

NEK2 deletion reduces chemotherapeutic resistance of CSCs. Chemotherapeutic resistance is an important characteristic of CSCs, which are known to show strong resistance to chemotherapy, thus, we examined 5-Fu and cisplatin resistance of HCC cells for NEK2 inhibition. Compared with control cells, NEK2 knockdown cells displayed significantly higher sensitivity to $5-\mathrm{Fu}$ and cisplatin chemotherapeutic agents in HCCLM3 $(\mathrm{P}<0.01$; Fig. 6A) and Hep-3B cells $(\mathrm{P}<0.01$; Fig. 6B). Finally, to investigate the mechanism responsible for 5-Fu and cisplatin resistance, we analyzed the mRNA expressions of multidrug resistant genes (ABCG2) and aldehyde 
dehydrogenase 1 family, member A1 Aliases (ALDH1A1). QRT-PCR showed that ABCG2 and ALDH1A1 were lowered in siNEK2 HCC cells (all $\mathrm{P}<0.01$; Fig. $6 \mathrm{C}$ and $\mathrm{D}$ ). All together, these data indicate that NEK2 may influence chemotherapeutic resistance through regulating the expression of ABCG2 and ALDH1A1 in HCC cells.

\section{Discussion}

The association between aberrant NEK2 expression and the prognosis of patients with HCC has not been previously reported. However, a few studies have estimated the impact of NEK2 expression on the prognosis of several other types of cancers. In the present study, we firstly demonstrated that increased expression of NEK2 was significantly associated with poor prognosis and was an independent prognostic factor in patients with HCC. The effects of NEK2 expression on CSC-like properties have not been examined in $\mathrm{HCC}$ or other cancers. In addition, our results from in vitro experiments indicated that knockdown of NKE2 expression contributed to the inhibition of CSC-like properties in $\mathrm{HCC}$, including the self-renewal and chemotherapeutic resistance properties.

CSC was first reported in acute myeloid leukemia (AML) (27), and subsequently found in some solid tumors, including HCC. NEK2 was associated with cancer cells in proliferation, drug resistance, apoptosis, tumorigenicity, invasion and migration $(11,28,29)$. To better elucidate the role of NEK2 in CSCs, we investigated the effect of NEK2 depletion on the self-renewal properties. As expected, the ability to form spheres and colonies was inhibited. Nanog, Sox 2 and Bmi-1 are three core transcription factors regulating cellular pluripotency and are known to suppress differentiation in ES cells $(4,30)$. CD133, EpCAM, K19, LIN28 and NOTCH1 which is frequently expressed in $\mathrm{HCC}$, has been predicted to be a CSC marker (31-34). Silencing NEK2 expression in HCC cells also downregulated the expression of stemness associated genes of CSCs, including Nanog, Sox2, Bmi-1, EpCAM, CD133, K19, LIN28 and NOTCH1.

The canonical Wnt/ $\beta$-catenin signaling pathway has emerged as a critical regulator of stem cells. In many tissues, activation of Wnt $/ \beta$-catenin signaling has also been associated with cancer. This has raised the possibility that the tightly regulated self-renewal mediated by $\mathrm{Wnt} / \beta$-catenin in stem and progenitor cells is converteded in cancer cells to allow malignant proliferation (35). Further investigation revealed that NEK2 influenced the self-renewal properties in HCC cells though the Wnt/ $\beta$-catenin signaling pathway. EpCAM and $\mathrm{c}-\mathrm{Myc}$ has been shown to be a direct transcriptional target in the Wnt/ $\beta$-catenin signaling pathway $(22,36)$, which has been suggested to play an important role in governing the self-renewal of cancer cells $(37,38)$. Our data indicated that the expression of $\beta$-catenin, EpCAM and c-Myc reduced accordingly when NEK2 was silenced. Furthermore, Wnt3a increased the ability of tumorsphere formation to partially compensate NEK2 inhibition. Taken together, these results suggest that the role of NEK2 in regulating self-renewal property is via activation of the Wnt/ $\beta$-catenin signaling pathway.

For HCC, traditional chemotherapeutic strategies do not completely eliminate tumors, which results in tumor recur- rence and drug resistance. The CSC model might explain this situation, and drug resistance is one of its properties $(39,40)$. We examined the influence of NEK 2 to drug resistance of HCC. Our results showed that NEK2 deletions were more sensitive to 5-Fu and cisplatin in HCC cells. This results was consistent with previous reports that NEK2 induced drug resistance in other cancers through different mechanisms, such as activation of efflux drug pumps (7) and regulation ALDH1A1-dependent drug resistance (41). Further qRT-PCR analyses revealed that knockdown of NEK2 decreased ABCG2 and ALDH1A1 level.

As an important multidrug resistance transporter, ABCG2 has the capability to efflux various chemotherapy drugs and contributes to drug resistance of cancer cells $(42,43)$. ABCG2 was also considered as a potential marker of CSCs in HCC and relevant with tumor stages and poor prognosis (44). ALDH1A1, belonged to the aldehyde dehydrogenases family of proteins, plays an important role in the metabolization of reactive acetaldehyde, and is expressed at high levels in stem cells and regulates stem cell function (45). Enforced expression of ALDH1A1 led to increased activity of the drug efflux pump to induce drug resistance (41). ALDH1A was also reported as a marker of several stem cancer cells (46-48). These findings further support the idea that NEK2 knockdown could increase the susceptibility of chemotherapy drugs and decrease the stemness marker for $\mathrm{HCC}$ by preferentially mediating ABCG2 and ALDH1A1.

In conclusion, we firstly demonstrated that upregulation of NEK2 expression contributed to poor prognosis in patients with HCC. Furthermore, the study also revealed the role of NEK2 in maintaining self-renewal property by means of Wnt/ $\beta$-catenin signaling, and influencing chemotherapeutic resistance by preferential regulating the expression of ABCG2 and ALDH1A1 in HCC cells. The present study provides a foundation for future studies that hold great promise for the development of a novel clinical biomarker for prediction of the malignant potential of $\mathrm{HCC}$, and therapeutic strategies for HCC patients.

\section{Acknowledgements}

The present study is financially supported by grants from the Health and Family Planning Commission of Zhejiang Province (grant nos. 2014KYB138 and 2015RCB014), and the National Natural Science Foundation of China (grant no. C0707).

\section{References}

1. Giordano S and Columbano A: MicroRNAs: New tools for diagnosis, prognosis, and therapy in hepatocellular carcinoma? Hepatology 57: 840-847, 2013.

2. Torre LA, Bray F, Siegel RL, Ferlay J, Lortet-Tieulent J and Jemal A: Global cancer statistics, 2012. CA Cancer J Clin 65: 87-108, 2015.

3. Poon D, Anderson BO, Chen LT, Tanaka K, Lau WY, Van Cutsem E, Singh H, Chow WC, Ooi LL, Chow P, et al; Asian Oncology Summit: Management of hepatocellular carcinoma in Asia: Consensus statement from the Asian Oncology Summit 2009. Lancet Oncol 10: 1111-1118, 2009.

4. Sainz B Jr and Heeschen C: Standing out from the crowd: Cancer stem cells in hepatocellular carcinoma. Cancer Cell 23: 431-433, 2013.

5. Wang B and Jacob ST: Role of cancer stem cells in hepatocarcinogenesis. Genome Med 3: 11, 2011. 
6. Wu W, Baxter JE, Wattam SL, Hayward DG, Fardilha M, Knebel A, Ford EM, da Cruz e Silva EF and Fry AM: Alternative splicing controls nuclear translocation of the cell cycle-regulated Nek2 kinase. J Biol Chem 282: 26431-26440, 2007.

7. Zhou W, Yang Y, Xia J, Wang H, Salama ME, Xiong W, Xu H, Shetty S, Chen T, Zeng Z, et al: NEK2 induces drug resistance mainly through activation of efflux drug pumps and is associated with poor prognosis in myeloma and other cancers. Cancer Cell 23: 48-62, 2013

8. Neal CP, Fry AM, Moreman C, McGregor A, Garcea G, Berry DP and Manson MM: Overexpression of the Nek2 kinase in colorectal cancer correlates with beta-catenin relocalization and shortened cancer-specific survival. J Surg Oncol 110 828-838, 2014

9. Takahashi Y, Iwaya T, Sawada G, Kurashige J, Matsumura T, Uchi R, Ueo H, Takano Y, Eguchi H, Sudo T, et al: Up-regulation of NEK2 by microRNA-128 methylation is associated with poor prognosis in colorectal cancer. Ann Surg Oncol 21: 205-212, 2014.

10. Wang S, Li W, Lv S, Wang Y, Liu Z, Zhang J, Liu T and Niu Y: Abnormal expression of Nek2 and $\beta$-catenin in breast carcinoma: Clinicopathological correlations. Histopathology 59: 631-642, 2011.

11. Marina M and Saavedra HI: Nek2 and Plk4: Prognostic markers, drivers of breast tumorigenesis and drug resistance. Front Biosci (Landmark Ed) 19: 352-365, 2014.

12. Zhong X, Guan X, Dong Q, Yang S, Liu W and Zhang L: Examining Nek2 as a better proliferation marker in non-small cell lung cancer prognosis. Tumour Biol 35: 7155-7162, 2014.

13. Bahmanyar S, Kaplan DD, Deluca JG, Giddings TH Jr, O'Toole ET, Winey M, Salmon ED, Casey PJ, Nelson WJ and Barth AI: beta-Catenin is a Nek2 substrate involved in centrosome separation. Genes Dev 22: 91-105, 2008.

14. Mbom BC, Siemers KA, Ostrowski MA, Nelson WJ and Barth AI: Nek2 phosphorylates and stabilizes $\beta$-catenin at mitotic centrosomes downstream of Plk1. Mol Biol Cell 25: 977-991, 2014

15. Chen L, Yuan YF, Li Y, Chan TH, Zheng BJ, Huang J and Guan XY: Clinical significance of CHD1L in hepatocellular carcinoma and therapeutic potentials of virus-mediated CHD1L depletion. Gut 60: 534-543, 2011.

16. Chen D, Xing W, Hong J, Wang M, Huang Y, Zhu C, Yuan Y and Zeng W: The beta2-adrenergic receptor is a potential prognostic biomarker for human hepatocellular carcinoma after curative resection. Ann Surg Oncol 19: 3556-3565, 2012.

17. Wang S, Li W, Liu N, Zhang F, Liu H, Liu F, Liu J, Zhang T and Niu Y: Nek2A contributes to tumorigenic growth and possibly functions as potential therapeutic target for human breast cancer. J Cell Biochem 113: 1904-1914, 2012

18. Wurmbach E, Chen YB, Khitrov G, Zhang W, Roayaie S, Schwartz M, Fiel I, Thung S, Mazzaferro V, Bruix J, et al: Genome-wide molecular profiles of HCV-induced dysplasia and hepatocellular carcinoma. Hepatology 45: 938-947, 2007.

19. Roessler S, Jia HL, Budhu A, Forgues M, Ye QH, Lee JS Thorgeirsson SS, Sun Z, Tang ZY, Qin LX, et al: A unique metastasis gene signature enables prediction of tumor relapse in early-stage hepatocellular carcinoma patients. Cancer Res 70: 10202-10212, 2010

20. Dravid G, Ye Z, Hammond H, Chen G, Pyle A, Donovan P, Yu X and Cheng L: Defining the role of Wnt/beta-catenin signaling in the survival, proliferation, and self-renewal of human embryonic stem cells. Stem Cells 23: 1489-1501, 2005.

21. Sato N, Meijer L, Skaltsounis L, Greengard P and Brivanlou AH: Maintenance of pluripotency in human and mouse embryonic stem cells through activation of Wnt signaling by a pharmacological GSK-3-specific inhibitor. Nat Med 10: 55-63, 2004.

22. Yamashita T, Budhu A, Forgues M and Wang XW: Activation of hepatic stem cell marker EpCAM by Wnt-beta-catenin signaling in hepatocellular carcinoma. Cancer Res 67: 10831-10839, 2007.

23. Yang W, Yan HX, Chen L, Liu Q, He YQ, Yu LX, Zhang SH, Huang DD, Tang L, Kong XN, et al: Wnt/beta-catenin signaling contributes to activation of normal and tumorigenic liver progenitor cells. Cancer Res 68: 4287-4295, 2008.

24. Monga SP: $\beta$-catenin signaling and roles in liver homeostasis, injury, and tumorigenesis. Gastroenterology 148: 1294-1310, 2015.

25. Pan W, Choi SC, Wang H, Qin Y, Volpicelli-Daley L, Swan L, Lucast L, Khoo C, Zhang X, Li L, et al: Wnt3a-mediated formation of phosphatidylinositol 4,5-bisphosphate regulates LRP6 phosphorylation. Science 321: 1350-1353, 2008.
26. Zhang KL, Han L, Chen LY, Shi ZD, Yang M, Ren Y, Chen LC, Zhang JX, Pu PY and Kang CS: Blockage of a miR-21/EGFR regulatory feedback loop augments anti-EGFR therapy in glioblastomas. Cancer Lett 342: 139-149, 2014.

27. Bonnet D and Dick JE: Human acute myeloid leukemia is organized as a hierarchy that originates from a primitive hematopoietic cell. Nat Med 3: 730-737, 1997.

28. Cappello P, Blaser H, Gorrini C, Lin DC, Elia AJ, Wakeham A, Haider S, Boutros PC, Mason JM, Miller NA, et al: Role of Nek2 on centrosome duplication and aneuploidy in breast cancer cells. Oncogene 33: 2375-2384, 2014.

29. Naro C, Barbagallo F, Chieffi P, Bourgeois CF, Paronetto MP and Sette C: The centrosomal kinase NEK2 is a novel splicing factor kinase involved in cell survival. Nucleic Acids Res 42: 3218-3227, 2014

30. Pei D: Regulation of pluripotency and reprogramming by transcription factors. J Biol Chem 284: 3365-3369, 2009.

31. Ma S, Chan KW, Hu L, Lee TK, Wo JY, Ng IO, Zheng BJ and Guan XY: Identification and characterization of tumorigenic liver cancer stem/progenitor cells. Gastroenterology 132: 2542-2556, 2007.

32. Yamashita T, Honda M, Nakamoto Y, Baba M, Nio K, Hara Y, Zeng SS, Hayashi T, Kondo M, Takatori H, et al: Discrete nature of $\mathrm{EpCAM}^{+}$and $\mathrm{CD} 90^{+}$cancer stem cells in human hepatocellular carcinoma. Hepatology 57: 1484-1497, 2013.

33. Kawai T, Yasuchika K, Ishii T, Katayama H, Yoshitoshi EY, Ogiso S, Kita S, Yasuda K, Fukumitsu K, Mizumoto M, et al: Keratin 19, a cancer stem cell marker in human hepatocellular carcinoma. Clin Cancer Res 21: 3081-3091, 2015.

34. Zheng YW, Nie YZ and Taniguchi H: Cellular reprogramming and hepatocellular carcinoma development. World J Gastroenterol 19: 8850-8860, 2013

35. Reya T and Clevers H: Wnt signalling in stem cells and cancer. Nature 434: 843-850, 2005

36. Kanwar SS, Yu Y, Nautiyal J, Patel BB and Majumdar AP: The Wnt/beta-catenin pathway regulates growth and maintenance of colonospheres. Mol Cancer 9: 212, 2010

37. Terris B, Cavard C and Perret C: EpCAM, a new marker for cancer stem cells in hepatocellular carcinoma. J Hepatol 52: 280-281, 2010.

38. Yamashita T, Ji J, Budhu A, Forgues M, Yang W, Wang HY, Jia H, Ye Q, Qin LX, Wauthier E, et al: EpCAM-positive hepatocellular carcinoma cells are tumor-initiating cells with stem/progenitor cell features. Gastroenterology 136: 1012-1024, 2009.

39. Hu X, Ghisolfi L, Keates AC, Zhang J, Xiang S, Lee DK and $\mathrm{Li}$ CJ: Induction of cancer cell stemness by chemotherapy. Cell Cycle 11: 2691-2698, 2012.

40. Dean M, Fojo T and Bates S: Tumour stem cells and drug resistance. Nat Rev Cancer 5: 275-284, 2005

41. Yang Y, Zhou W, Xia J, Gu Z, Wendlandt E, Zhan X, Janz S, Tricot G and Zhan F: NEK2 mediates ALDH1A1-dependent drug resistance in multiple myeloma. Oncotarget 5: 11986-11997, 2014.

42. Gottesman MM, Fojo T and Bates SE: Multidrug resistance in cancer: Role of ATP-dependent transporters. Nat Rev Cancer 2: 48-58, 2002.

43. Robey RW, To KK, Polgar O, Dohse M, Fetsch P, Dean M and Bates SE: ABCG2: A perspective. Adv Drug Deliv Rev 61: 3-13, 2009.

44. Zhang G, Wang Z, Luo W, Jiao H, Wu J and Jiang C: Expression of potential cancer stem cell marker ABCG2 is associated with malignant behaviors of hepatocellular carcinoma. Gastroenterol Res Pract 2013: 782581, 2013. doi:10.1155/2013/782581.

45. Koppaka V, Thompson DC, Chen Y, Ellermann M, Nicolaou KC, Juvonen RO, Petersen D, Deitrich RA, Hurley TD and Vasiliou V: Aldehyde dehydrogenase inhibitors: A comprehensive review of the pharmacology, mechanism of action, substrate specificity, and clinical application. Pharmacol Rev 64: 520-539, 2012.

46. Luo Y, Dallaglio K, Chen Y, Robinson WA, Robinson SE, McCarter MD, Wang J, Gonzalez R, Thompson DC, Norris DA, et al: ALDH1A isozymes are markers of human melanoma stem cells and potential therapeutic targets. Stem Cells 30: 2100-2113, 2012.

47. Balicki D: Moving forward in human mammary stem cell biology and breast cancer prognostication using ALDH1. Cell Stem Cell 1: 485-487, 2007.

48. Choi SA, Lee JY, Phi JH, Wang KC, Park CK, Park SH and Kim SK: Identification of brain tumour initiating cells using the stem cell marker aldehyde dehydrogenase. Eur J Cancer 50: 137-149, 2014. 